\title{
Variational Multiscale Methods for Incompressible Flows
}

\author{
V. Gravemeier, S. Lenz \& W.A. Wall \\ Chair for Computational Mechanics \\ Technische Universität München \\ Boltzmannstr. 15, D-85747 Garching b. München \\ http://www.lnm.mw.tum.de \\ (vgravem, lenz, wall) @lnm.mw . tum.de
}

\section{Introduction}

The numerical simulation of incompressible flow governed by the Navier-Stokes equations requires to deal with subgrid phenomena. Particularly in turbulent flows, the scale spectra are notably widened and need to be handled adequately to get a reasonable numerical solution. Separating the complete scale range into subranges enables a different treatment of any of these subranges. In the brevity of this paper, the idea and applications of two methods are sketched. In the corresponding talk, we will present the general framework of a two- and a three-scale separation of the incompressible Navier-Stokes equations based on the variational multiscale method as proposed in [9] and [3]. In a two-scale separation, resolved and unresolved scales are distinguished, and in a three-scale separation, large resolved scales, small resolved scales, and unresolved scales are differentiated. After having presented the general framework, three different approaches to numerical realizations will be addressed (i.e., a global, a local, and a new residual-based approach). A comprehensive overview may be found in [6].

\section{The variational multiscale framework}

The set of incompressible Navier-Stokes equations in conservative form is given as

$$
\begin{array}{rlrl}
\frac{\partial \mathbf{u}}{\partial t}+\nabla \cdot(\mathbf{u} \otimes \mathbf{u})-2 \nu \nabla \cdot \varepsilon(\mathbf{u})+\nabla p & =\mathbf{f} & & \text { in } \Omega \\
\nabla \cdot \mathbf{u} & =0 & \text { in } \Omega
\end{array}
$$

with appropriate initial and boundary conditions. Here, $\mathbf{u}$ and $p$ denote velocity and pressure, $\varepsilon(\mathbf{u})$ the rate-of-deformation tensor, $\nu$ the kinematic viscosity, and $\mathbf{f}$ a body force.

A weighted residual formulation of the Navier-Stokes equations is given as follows: find $\{\mathbf{u}, p\} \in \mathcal{S}_{\mathbf{u} p}$, such that

$$
B_{\mathrm{NS}}(\mathbf{v}, q ; \mathbf{u}, p)=(\mathbf{v}, \mathbf{f})_{\Omega} \quad \forall\{\mathbf{v}, q\} \in \mathcal{V}_{\mathbf{u} p},
$$

where $\mathbf{v}$ and $q$ denote the weighting functions. $\mathcal{S}_{\mathbf{u} p}$ and $\mathcal{V}_{\mathbf{u} p}$ represent the combined formulation of the solution and weighting function spaces for velocity and pressure: $\mathcal{S}_{\mathbf{u} p}:=\mathcal{S}_{\mathbf{u}} \times \mathcal{S}_{p}$ and $\mathcal{V}_{\mathbf{u} p}:=\mathcal{V}_{\mathbf{u}} \times \mathcal{V}_{p}$. The form $B_{\mathrm{NS}}(\mathbf{v}, q ; \mathbf{u}, p)$ on the left hand side of $(3)$ is defined as

$$
\begin{aligned}
B_{\mathrm{NS}}(\mathbf{v}, q ; \mathbf{u}, p)= & \left(\mathbf{v}, \frac{\partial \mathbf{u}}{\partial t}\right)_{\Omega}+(\mathbf{v}, \nabla \cdot(\mathbf{u} \otimes \mathbf{u}))_{\Omega}+(\mathbf{v}, \nabla p)_{\Omega} \\
& -(\mathbf{v}, 2 \nu \nabla \cdot \varepsilon(\mathbf{u}))_{\Omega}+(q, \nabla \cdot \mathbf{u})_{\Omega} .
\end{aligned}
$$

In a three-scale separation, the solution functions are separated as

$$
\mathbf{u}=\overline{\mathbf{u}}+\mathbf{u}^{\prime}+\hat{\mathbf{u}}, \quad p=\bar{p}+p^{\prime}+\hat{p},
$$


where $\overline{(\cdot)},(\cdot)^{\prime}$, and $(\hat{\cdot})$ denote the large resolved, small resolved, and unresolved scales, respectively. The weighting functions are separated accordingly. Due to the linearity of the weighting functions, the weighted residual equation (3) may now be decomposed into a system of three equations reading

$$
\begin{array}{cl}
B_{\mathrm{NS}}\left(\overline{\mathbf{v}}, \bar{q} ; \overline{\mathbf{u}}+\mathbf{u}^{\prime}+\hat{\mathbf{u}}, \bar{p}+p^{\prime}+\hat{p}\right)=(\overline{\mathbf{v}}, \mathbf{f})_{\Omega} & \forall(\overline{\mathbf{v}}, \bar{q}) \in \overline{\mathcal{V}}_{\mathbf{u} p} \\
B_{\mathrm{NS}}\left(\mathbf{v}^{\prime}, q^{\prime} ; \overline{\mathbf{u}}+\mathbf{u}^{\prime}+\hat{\mathbf{u}}, \bar{p}+p^{\prime}+\hat{p}\right)=\left(\mathbf{v}^{\prime}, \mathbf{f}\right)_{\Omega} & \forall\left(\mathbf{v}^{\prime}, q^{\prime}\right) \in \mathcal{V}_{\mathbf{u} p}^{\prime} \\
B_{\mathrm{NS}}\left(\hat{\mathbf{v}}, \hat{q} ; \overline{\mathbf{u}}+\mathbf{u}^{\prime}+\hat{\mathbf{u}}, \bar{p}+p^{\prime}+\hat{p}\right)=(\hat{\mathbf{v}}, \mathbf{f})_{\Omega} & \forall(\hat{\mathbf{v}}, \hat{q}) \in \hat{\mathcal{V}}_{\mathbf{u} p}
\end{array}
$$

Furthermore, it is assumed that the direct influence of the unresolved scales on the large resolved scales is close to zero, relying on a clear separation of the large-scale space and the space of unresolved scales. This leads to a simplified equation system.

It is not intended to resolve anything called unresolved a priori. Taking into account the effect of the unresolved scales onto the small scales is the only desire. Some alternatives lend themselves for this purpose, but the focus in the corresponding talk will be on the subgrid viscosity approach as a usual and well-established way of taking into account the effect of unresolved scales in the traditional LES. In the variational multiscale approach to LES, the subgrid viscosity term directly acts only on the small resolved scales. Indirect influence on the large resolved scales, however, is ensured due to the coupling of the large- and the small-scale equations.

\section{Practical methods}

At this stage, it should be pointed out that the variational multiscale method is a theoretical framework for the separation of scales. Thus, it is essential to develop corresponding practical implementations by incorporating the variational multiscale framework into a specific numerical method. For such practical methods, it is crucial that the aforementioned separation of the different scale groups is actually achieved. Implementations of the three-scale separation have already been done within continuous Galerkin finite element methods, discontinuous Galerkin finite element methods, finite volume methods, finite difference methods, and spectral methods, see [6]. In the following, it will be focused on the authors' work in this context, which aimed at finite element as well as finite volume methods.

We have started with a residual-free-bubble approach for the resolution of the small resolved scales, see, e.g., [7]. Since the underlying assumption (i.e., the mutual influence of the small scales is confined to individual elements of an initially chosen discretization) often appears to be too restrictive, we turned to an approach where the interaction of the small resolved scales is not localized in such a way, see [4]. This global variational multiscale approach was applied to turbulent flow in a channel in [4] and turbulent flow in a diffuser in [5]. As outlined above, three different scale groups (i.e., large resolved scales, small resolved scales, and unresolved scales) are distinguished, and the modeled effect of the unresolved scales is directly applied only to the small resolved scales. In contrast to the local approach based on residual-free bubbles, both large and small scales are globally solved for as part of a global equation system within the global approach. Various scale-separating operators as well as dynamic and constant-coefficient-based subgrid-scale modeling were investigated within a finite volume method. Excellent results were achieved, as documented in the aforementioned publications. However, a similar approach may also be applied, for instance, within a finite element method.

Recently, a new residual-based approach has been developed in [2] where only resolved and unresolved scales are distinguished (i.e., a two-scale separation). This new concept approxmiates the unresolved scales by analytical expressions. It may be considered an advanced stabilized method which takes into account the non-linearity of the Navier-Stokes equations. The influence of the unresolved scales on the resolved scales, with no further distinction between large and 


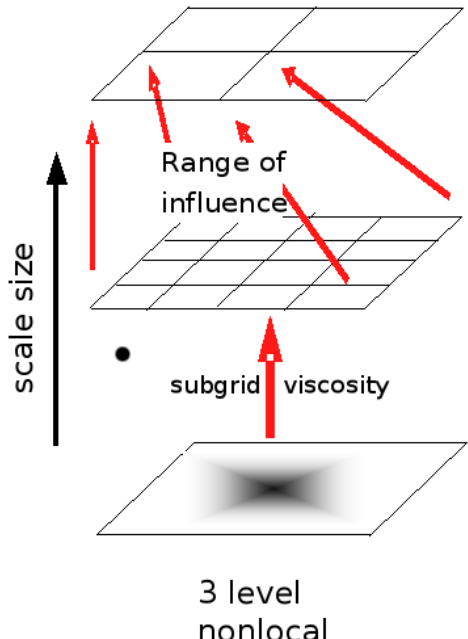

nonlocal

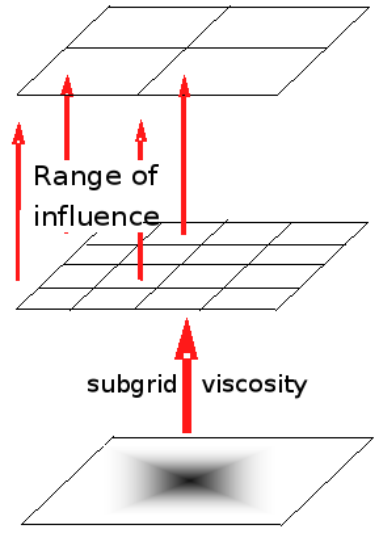

3 level

local

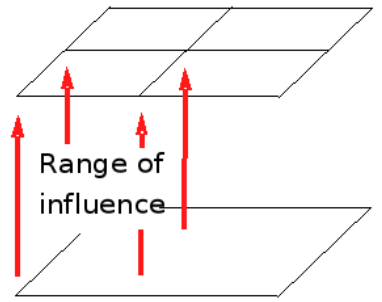

NewVMM

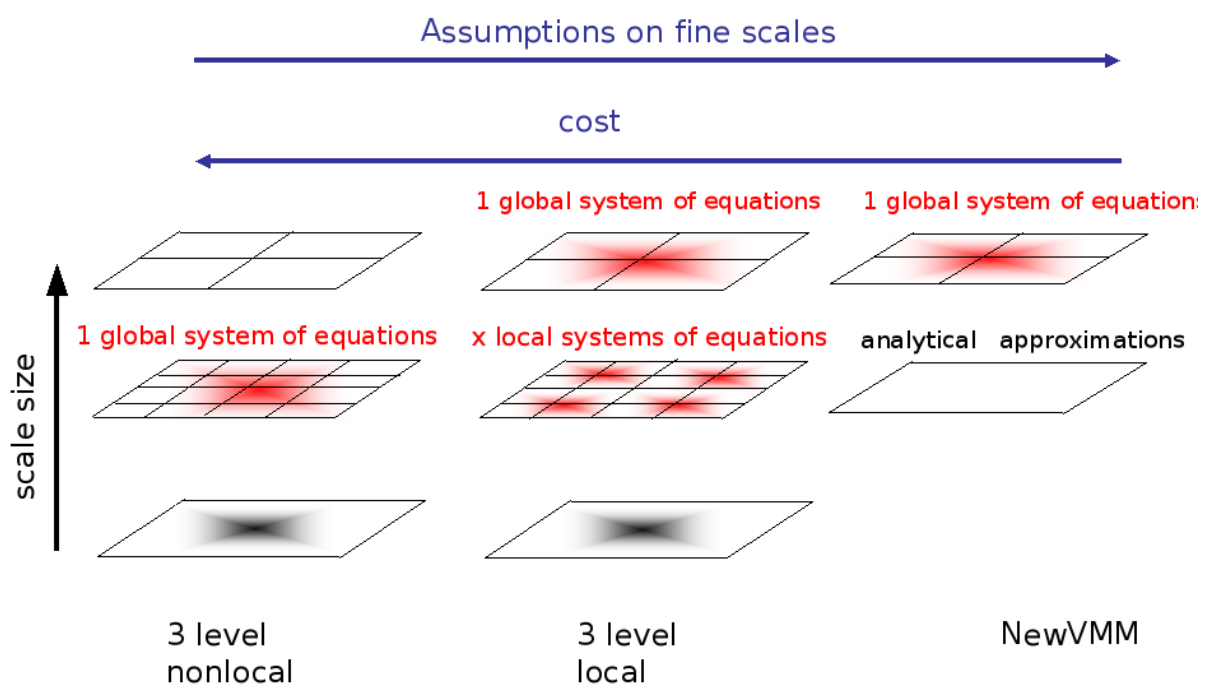

Figure 2: Systems of equations arising from different approaches

small resolved scales, is confined to individual elements of the discretization similar to the residual-free-bubble approach. This is due to the fact that the analytical approximations of the unresolved scales make use of stabilization parameters, which, in turn, are linked to element Green's functions and residual-free bubbles, see, e.g., [8]. Our results obtained with the residualbased approach are about to be published in [10].

The range of influence of the small scales for the aforementioned methods is depicted in Fig. 1, and the systems of equations to be solved are sketched in Fig. 2. In the following sections, numerical examples both for the application of the global approach to the variational multiscale method based on multigrid scale-separating operators as described in [4] and the new residual-based variational multiscale method will be described.

\section{Turbulent test case for the global approach to the variational multiscale method}

The multigrid-based global approach as addressed above was implemented into the CDP- $\alpha$ code, the flagship LES code of the Center for Turbulence Research (Stanford University and NASA Ames Research Center), as described in [4]. Underlying this code is a second-order accurate energy-conserving finite volume method particularly suited for applications on unstructured 


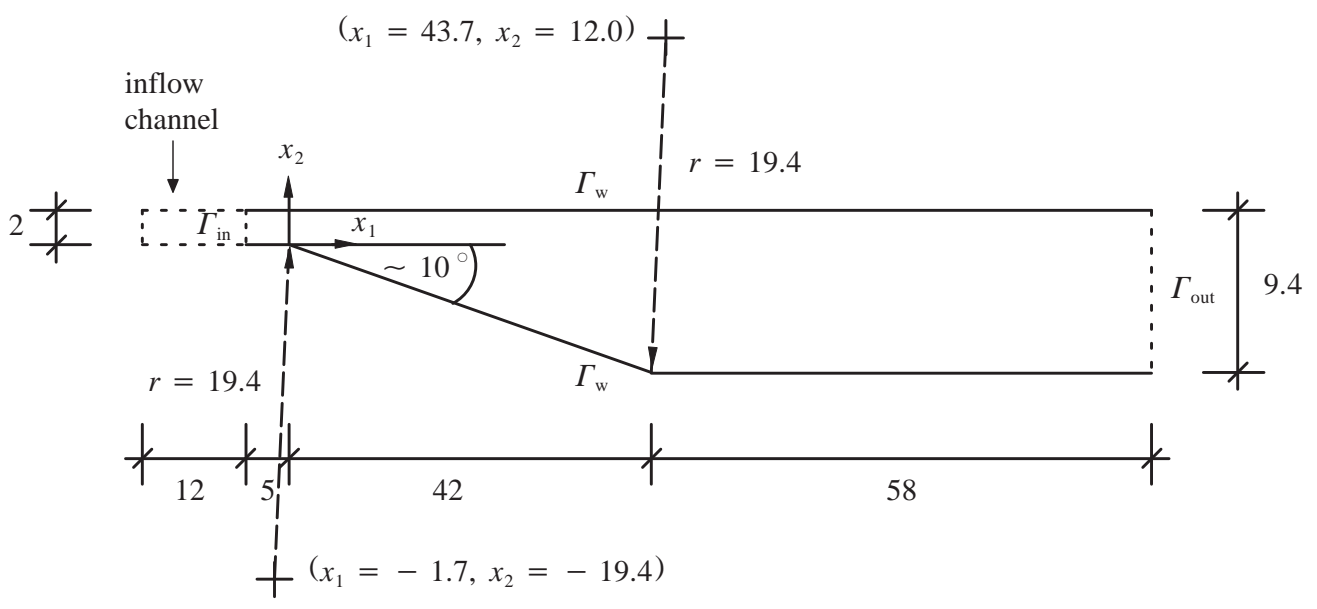

Figure 3: Diffuser geometry in $x_{1}-x_{2}$-plane

grids.

The method was applied to turbulent flow in a planar asymmetric diffuser as a challenging test case. Turbulent flow in such a diffuser is a representative of the group of flow problems exhibiting more than one direction of inhomogeneity. Not only for this reason, it is a more challenging flow problem. Several features of this flow indicate its higher complexity (i.e., a large unsteady separation bubble due to an adverse pressure gradient, a sudden change of the streamwise pressure gradient from slightly favorable to strongly adverse at the diffuser throat, and a slowly growing internal layer emerging at the upper flat wall in the relaxation zone downstream of the sharp variation in the streamwise pressure gradient). The problem setup and several results from this test case are reported in [5]. The diffuser geometry, which basically matches the experimental configuration in [1] ("Buice-experiment") as well as the numerical setup in [11] ("Wu-LES"), is shown in Fig. 3. Some remarks on the efficiency of the method as well as some selected results in comparison to the aforementioned experimental and LES results in [1] and [11], respectively, will be given below.

Three different methods are investigated: the dynamic Smagorinsky (DS) model in a nonmultiscale application, the constant-coefficient-based Smagorinsky model within the multiscale environment (CMS), and the dynamic Smagorinsky model within the multiscale environment (DMS). All of these methods are analyzed for a projective scale-separating operator $S^{\mathrm{pm}}$ as described in [4]. The abbreviation "DMS-PM", for instance, indicates the variational multiscale LES incorporating a dynamic Smagorinsky model, with the scale-separating operator $S^{\mathrm{pm}}$ applied. The non-projective scale-separating operator $S^{\mathrm{sm}}$ (see [4]) is only investigated for CMS, since this method revealed the most notable differences between the scale-separating operators for turbulent channel flow in [4]. Results are also reported for simulations using no model at all (NM), which represents a coarse (i.e., not sufficiently resolved) DNS. The LES in [11], which the results are compared to, applied the same dynamic Smagorinsky model in a traditional non-multiscale LES (i.e., DS using smooth filters for scale separation).

Evaluating the necessary computational effort provides the following numbers. Setting the computational effort for NM to 1.0, the relative measures for CMS-PM, CMS-SM, DS-PM and DMS-PM are approximately 1.08, 1.34, 1.27, and 1.32, respectively. These numbers are even more impressively in favor of CMS-PM than the ones for the channel in [4]. Thus, it is confirmed that CMS in combination with PM is a very efficient method computationally, in the present case substantially more efficient than, for instance, DS. Using the scale-separating operator SM, the numbers increase drastically for CMS. Less effort is required for PM compared to SM for reasons explained in [4] and [5].

As one sample of the flow parameters investigated, Fig. 4 depicts the results for the skin 


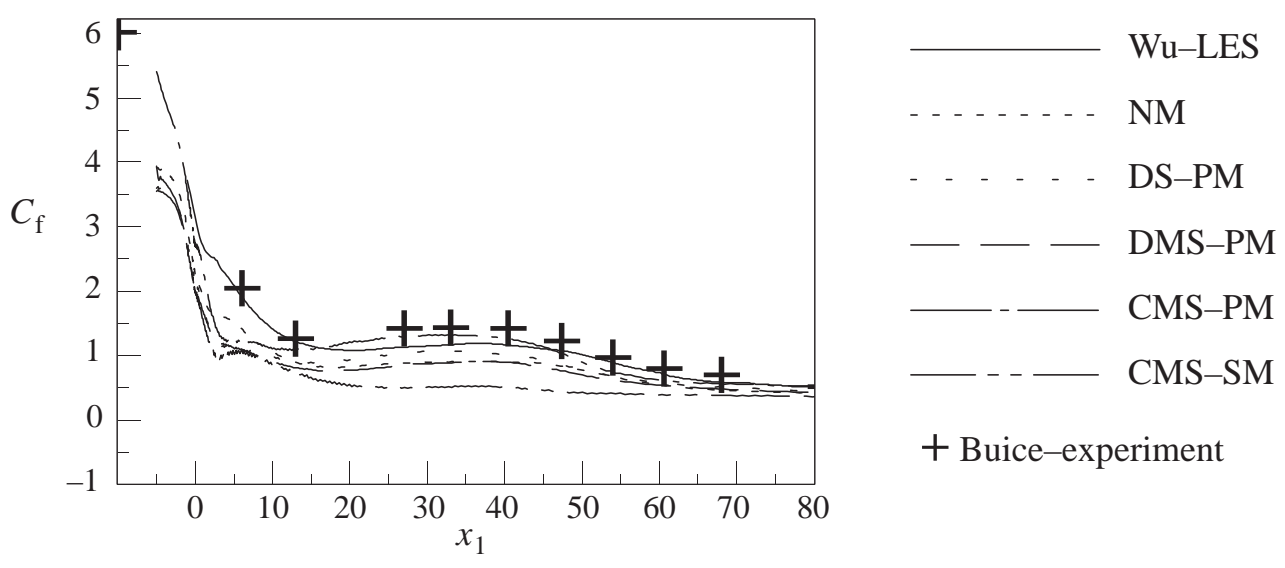

Figure 4: Skin friction coefficient (factor 1000) along the upper wall of the diffuser

friction coefficient along the upper wall of the diffuser. Results for further flow parameters can be found in [5]. It is stated that all methods tend to underpredict $C_{\mathrm{f}}$ compared to the results from the Wu-LES and the Buice-experiment. The worst results are produced by CMS-SM. The profile for NM is closest to the ones from the Wu-LES and the Buice-experiment immediately behind the diffuser throat, but gets worse in its prediction further downstream. DS-PM yields a fairly good prediction throughout the diffuser, and DMS-PM produces worse results than DSPM. Although the results for CMS-PM are worse than the ones for NM immediately behind the diffuser throat, the predicition is the best overall. It is the only method yielding results which almost match the experimental results in the section of the diffuser between $x_{1} \approx 18$ and $x_{1} \approx 46$. In this part of the diffuser, which is approximately the region where the flow is separated, CMSPM appears to produce even better results than the substantially finer discretized Wu-LES. Furthermore, it seems to be the only one of the present methods which would have been able to predict the first point from the Buice-experiment at $x_{1} \approx-10$, if the inlet channel had been elongated.

\section{Turbulent test case for the residual-based variational multiscale method}

The residual-based variational multiscale method (RBVMM) is applied to turbulent flow in a channel at Reynolds number $R e_{\tau}=180$. The simulations are performed with a resolution of 33 nodes in all three spatial directions. The mesh is stretched in wall-normal direction in form of a cosine function, in order to resolve the boundary layer. Periodic boundary conditions for velocity and pressure are applied at the boundaries in streamwise $x_{1}$-direction as well as in spanwise $x_{3}$-direction. Various LES results obtained with three different Lagrangean finite element basis functions are compared to DNS results. VMM8 denotes the aforementioned RBVMM approach using linear basis functions yielding 8-noded hexahedra in three dimensions. Moreover, the 20-noded quadratic serendipity element (VMM20) and the 27-noded complete quadratic element (VMM27) based on this approach are investigated. For comparison, an AGLS-stabilized approach using the 20-noded serendipity element (AGLS20) is utilized.

Concerning computational cost, there is virtually no difference between the RBVMM approach and the AGLS approach, since the evaluation of the additional element matrices for the RBVMM approach is cheap. In contrast, there are significant differences between the cost for linear and quadratic elements. For linear elements, no expensive second derivatives have to be calculated, and moreover, the solution of the resulting linear system of equations is cheaper than that of the quadratic elements. As a rule-of-thumb, for our iterative solver, we have found a factor of roughly six relating the cost for linear 8-noded to the cost for the quadratic 27-noded hexahedra. Moreover, due to the possibility to use a time step which is twice as large for the 


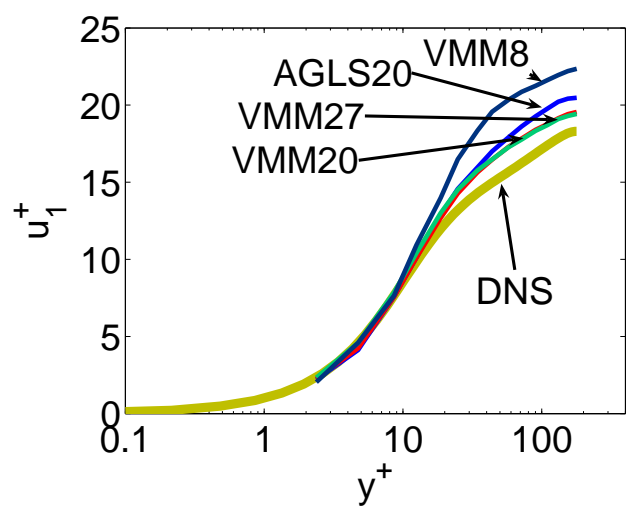

Figure 5: Mean streamwise velocity

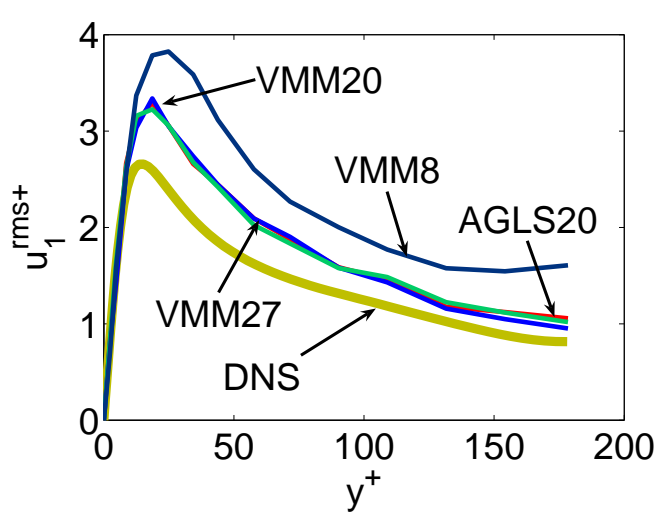

Figure 6: Rms of velocity fluctuations in streamwise direction

quadratic elements than for the linear elements, the transitioning to a statistically steady state takes less time steps for the quadratic elements. For an element size corresponding to 33 nodes in all spatial directions, the quadratic 20-noded serendipity elements save about half the number of degrees of freedom. Thus, their cost is approximately half of that for the quadratic 27-noded elements (i.e., about three times the effort for the linear elements).

In Fig. 5, the normalized mean streamwise velocity $u_{1}^{+}$is displayed dependent on wall coordinates $y^{+}$. An enormous improvement can be observed for quadratic elements AGLS20, VMM20, and VMM27 in comparison to the linear elements VMM8. Comparing VMM8 and AGLS20, it becomes obvious that elevating the interpolation order has a more significant impact on the accuracy of the results than introducing the RBVMM. Focussing on the quadratic elements, this plot shows that the RBVMM yields significantly better results than the classical stabilization approach. The results for the serendipity VMM20 and the complete quadratic VMM27 elements are more or less the same.

With respect to the velocity fluctuations in all three spacial directions (Figs. 6, 7, and 8), the observations described above still hold. Quadratic elements perform better than linear elements even without the additional RBVMM terms. Comparing quadratic elements, the introduction of the additional RBVMM terms does not lead to significantly better results for the velocity fluctuations. What is noticeable are the oscillations in the results for quadratic serendipity elements VMM20 and AGLS20 in Figs. 7 and 8, and for the full quadratic elements VMM27 in Fig. 8. An explanation might be the abidance of different continuity conditions of interior and corner nodes for Lagrangean finite elements. Apart from this, VMM27 elements yield better results for the spanwise velocity fluctuations near the boundary and behave comparably to VMM20 elements towards the channel center, see Fig. 8. Concerning the velocity fluctuations in wall-normal direction (see Fig. 7), significantly different results of VMM27 elements and VMM20 elements are observable. Near the wall, VMM27 elements perform worse than VMM20 elements, and towards the channel center, they yield significantly better results.

\section{Acknowledgements}

Support through the Deutsche Forschungsgemeinschaft (DFG), the Center for Turbulence Research (Stanford University and NASA Ames Research Center), and the Alexander von HumboldtFoundation is gratefully acknowledged. 


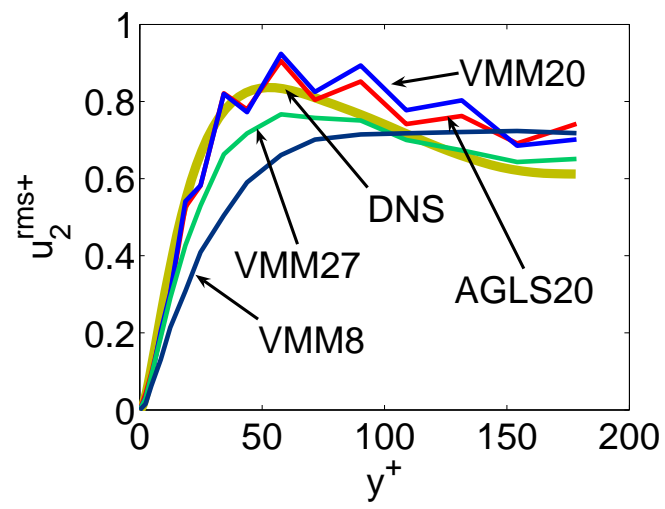

Figure 7: Rms of velocity fluctuations in wall-normal direction

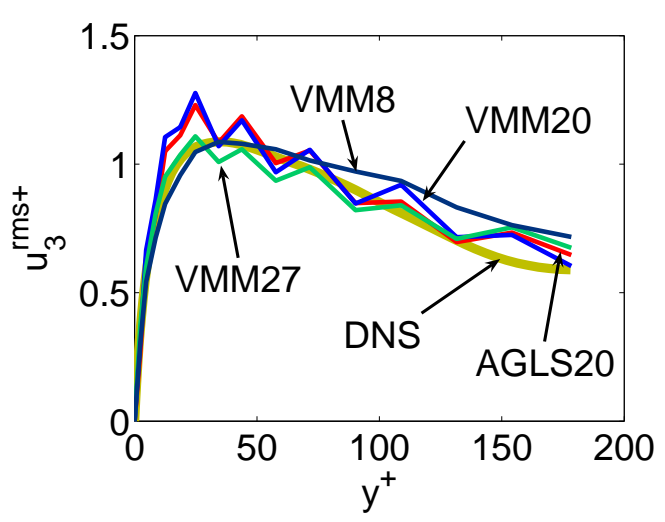

Figure 8: Rms of velocity fluctuations in spanwise direction

\section{References}

[1] C.U. Buice and J.K. Eaton, "Experimental investigation of flow through an asymmetric plane diffuser", TSD-10\%, Department of Mechanical Engineering, Stanford University, 1997.

[2] V.M. Calo, "Residual-based multiscale turbulence modeling: finite volume simulations of bypass transition", PhD Thesis, Department of Civil and Environmental Engineering, Stanford University, 2005.

[3] S.S. Collis, "Monitoring unresolved scales in multiscale turbulence modeling", Physics of Fluids, 13, 1800-1806 (2001).

[4] V. Gravemeier, "Scale-separating operators for variational multiscale large eddy simulation of turbulent flows", Journal of Computational Physics, 212, 400-435 (2006).

[5] V. Gravemeier, "Variational multiscale large eddy simulation of turbulent flow in a diffuser", Computational Mechanics, in press, 2006.

[6] V. Gravemeier, "The variational multiscale method for laminar and turbulent flow", Archives of Computational Methods in Engineering, in press, 2006.

[7] V. Gravemeier, W.A. Wall and E. Ramm, "Large eddy simulation of turbulent incompressible flows by a three-level finite element method", International Journal for Numerical Methods in Fluids, 48, 1067-1099 (2005).

[8] T.J.R. Hughes, G.R. Feijoo, L. Mazzei and J.-B. Quincy, "The variational multiscale method-a paradigm for computational mechanics", Computer Methods in Applied Mechanics and Engineering, 166, 3-24 (1998).

[9] T.J.R. Hughes, L. Mazzei and K.E. Jansen, "Large eddy simulation and the variational multiscale method", Computing and Visualization in Science, 3, 47-59 (2000).

[10] S. Lenz and W.A. Wall, "A residual-based variational multiscale method and its application to turbulent channel flow", submitted to Theoret. Comput. Fluid Dyn., 2006.

[11] X. Wu, J. Schlüter, P. Moin, H. Pitsch, G. Iaccarino and F. Ham, "Computational study on the internal layer in a diffuser", Journal of Fluid Mechanics, 550, 391-412 (2006). 